\title{
Intracranial Meningioma with Polygonal Granular Cell Appearance in a Chihuahua
}

\author{
Yoshinori TAKEUCHI ${ }^{1)}$, Yumi OHNISHI ${ }^{2)}$, Satoru MATSUNAGA ${ }^{2)}$, Hiroyuki NAKAYAMA ${ }^{1)}$ and Koji UETSUKA ${ }^{1)}$ \\ ${ }^{1)}$ Departments of Veterinary Pathology and ${ }^{2)}$ Veterinary Clinical Science, Graduate School of Agricultural and Life Sciences, \\ The University of Tokyo, 1-1-1 Yayoi, Bunkyo-ku, Tokyo 113-8657, Japan
}

(Received 27 August 2007/Accepted 17 January 2008)

ABSTRACT. A menigioma with polygonal granular cell proliferation in an 11-year and 8-month-old male Chihuahua is described. The tumor was observed under the dura matter of the right cerebrum. Microscopically, the tumor consisted of solid growth foci of small- or large- sized polygonal cells, with pale-stained nuclei, prominent nucleoli, and fine granular to foamy eosinophilic cytoplasm. Some of the proliferating cells contained variable amounts of cytoplasmic PAS-positive granules. Immunohistochemical analysis revealed that neoplastic cells were positive for vimentin and S-100 protein. Ultrastructurally, the neoplastic cells contained vesicular structures with a few small round-shaped bodies in the cytoplasm. We diagnosed the case as canine meningioma with granular cell appearance. KEY WORDS: canine, granular cell, meningioma.

Meningiomas are derived from meningothelial cells of the arachnoid membrane and pia matter of the nervous system [4], and are common in mature dogs $[12,13]$. Intracranial meningiomas occur in the meninges not only over the convexities, attached to the cerebral falx, below the brain stem, and attached to the cerebellar tentorium, but at an intraventricular location associated with the choroid plexus, and in rare cases at retrobulbar locations $[6,13]$. The most recent classification of meningiomas in domestic animals describes nine histological types: meningothelial, fibrous, transitional, psammomatous, angiomatous, papillary, granular cell, myxoid and anaplastic [3]. Among these types, granular cell meningioma is specific to domestic animals, and does not exist in the WHO classification of human meningioma [5]. However, there have been only a few reports of the type of meningioma with granular cell components in dogs $[9,10]$.

There are some reports of canine granular cell tumors in the meninges $[2,8,11]$. At present, the histopathological differences between granular cell meningiomas and granular cell tumors in the brain are obscure. Furthermore, the description of the histological characteristics of granular cell meningioma in the WHO classification for domestic animals is very similar to that of granular cell tumors, though they are considered to be different tumors in dogs. Meningioma is certainly derived from meningothelial cells, while the histogenesis of granular cell tumors in dogs remains controversial.

We report here a case of intracranial neoplasia with proliferation of polygonal granular cells in a Chihuahua.

An 11-year and 8-month-old male Chihuahua dog presented at the Veterinary Medical Center, the University of Tokyo, with a complaint of paresis of the left forelimb and

\footnotetext{
* Correspondence to: Takeuchi, Y., Departments of Veterinary Pathology, Graduate School of Agricultural and Life Sciences, The University of Tokyo, 1-1-1 Yayoi, Bunkyo-ku, Tokyo 1138657, Japan.

e-mail: ytake-tky@umin.net
}

seizure. The magnetic resonance image (MRI) examination revealed an expanded lesion that draped the right cerebrum. The dog was diagnosed with chronic meningoencephalitis, mesencephalic arachnoid cyst and syringomyelia, and then treated with steroidal anti-inflammatory drugs, anticonvulsants and antibiotics. Although the animal was well for a short period after the treatment, he suddenly developed a strong seizure and an astasia 8 months later, and died of aspiration pneumonia and respiratory failure.

At autopsy, a gray-white-colored rough membranous structure of 1 to $2 \mathrm{~mm}$ thickness was observed under the dura matter of the right cerebrum. The structure was widely spread over the parietal and temporal lobes and basis cerebri, and attached to the dura matter of the parietal lobe as well. The left lateral ventricle was dilated.

The brain was fixed in $10 \%$ neutral-buffered formalin, and embedded in paraffin wax and cut into $4-\mu \mathrm{m}$-thick sections. Deparaffinized sections were stained with hematoxylin and eosin (HE). The sections were also stained by diastase-resistant periodic acid-Shiff (PAS) staining. Oil Red-O stain and Sudan Black B stain were performed on unprocessed, 4- $\mu \mathrm{m}$-thick, formalin-fixed, frozen tissue. Immunohistochemical examination was carried out by the labeled streptavidin biotinylated antibody (LSAB) method, and with antibodies against the following proteins: vimentin (1:50; Dako, Carpinteria, CA); S-100 protein (1:50; Dako); cytokeratin (Wide Spectrum Screening) (1:50; Dako); lysozyme (1:50; Dako); desmin (1:50; Dako); neuron specific enolase (NSE, 1:50; Dako); glial fibrillary acidic protein (GFAP, 1:50; Dako); myeloid/histiocyte Antigen (MAC387, 1:50; Dako); and HLA-DR (1:50; Dako). Small pieces of the membranous tissue were subjected to electron microscopic examination. Briefly, the tissue was refixed in glutaldehyde and osmium tetroxide, and embedded in Epon 812 using a routine procedure. Ultrathin sections were double-stained with uranyl acetate and lead citrate, and observed using a JEM 1200 electron microscope (JEOL Ltd., Tokyo, Japan) at $80 \mathrm{KV}$. 

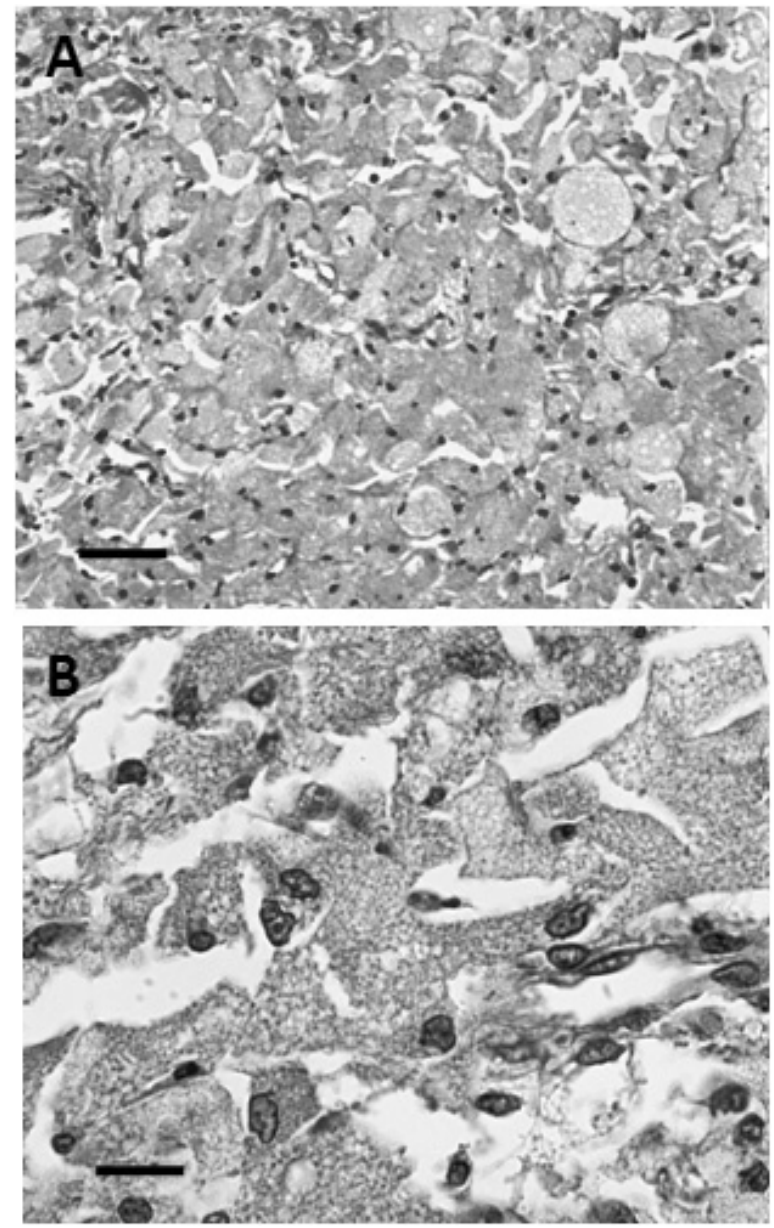

Fig. 1. Polygonal neoplastic cells with fine granular to foamy eosinophilic cytoplasm. HE. Bar, $50 \mu \mathrm{m}$ (A), and $10 \mu \mathrm{m}$ (B).

Histopathological examination revealed that the membranous structure consisted of solid growth foci of small- or large- sized polygonal cells. There was a thick collagenous fiber stratum between the growth foci and the cerebral cortex, suggesting that the growth foci were located in the meninges. The proliferating polygonal cells showed the fine granular to foamy eosinophilic cytoplasm (Fig. 1). Their nuclei were pale-stained and had prominent nucleoli. Although mitotic figures were rare, proliferating cells invaded into the cerebral parenchyma in part (Fig. 2). In the growth foci, small necrotic regions were randomly distributed, and a small number of concentric psammoma bodies were observed (Fig. 3). Aggregated infiltration of lymphocytes and plasma cells in pial perivascular areas in the cerebral parenchyma was also observed. Neuronal cell atrophy, neuronophagia, gliosis and gitter cell infiltration were observed in the cerebral parenchyma adjacent to the neoplastic lesion. No similar proliferating lesions were observed in other organs.

In PAS stain, cytoplasmic granules of the proliferating cells were stained very weakly, which was diastase-resistant

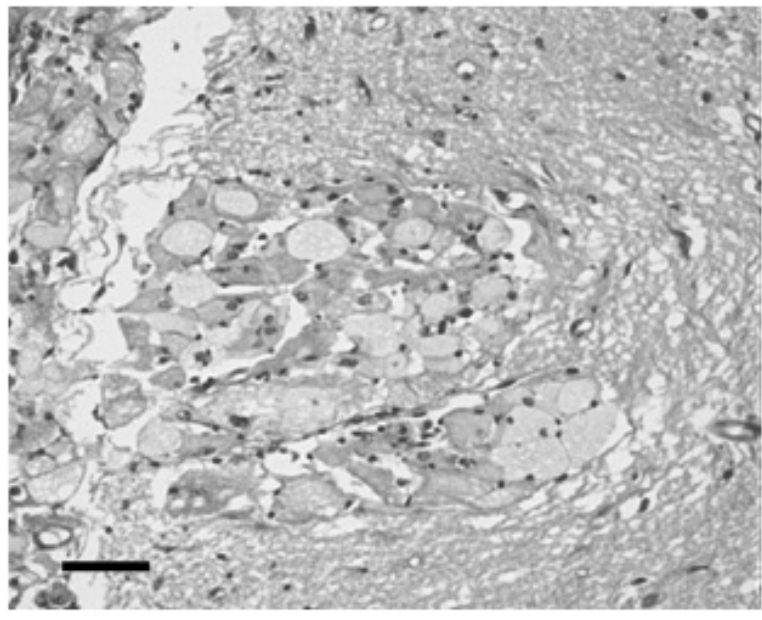

Fig. 2. Neoplastic cells invading cerebral parenchyma. HE. Bar, $50 \mu \mathrm{m}$.

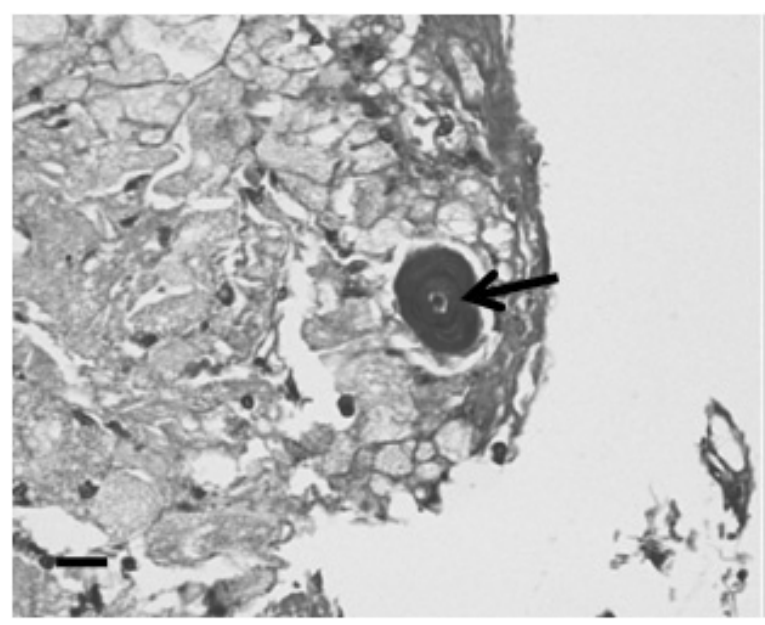

Fig. 3. A concentric psammoma body (arrow). HE. Bar, $20 \mu \mathrm{m}$.

(Fig. 4). The cytoplasmic vacuoles of proliferating cells were Oil-Red O and Sudan Black B negative. Most of the neoplastic cells were diffusely and strongly positive for vimentin and S-100 protein (Fig. 5), and were negative for cytokeratin, lysozyme, desmin, GFAP and MAC387. Only a small number of the cells were positive for NSE and HLADR.

Ultrastructurally, the neoplastic cells contained vesicular structures with a few small round-shaped bodies in the cytoplasm (Fig. 6). However, the structures and bodies were distributed very sparsely, and it was difficult to identify the origin of the organelles. Other cytoplasmic organelles were not observed in neoplastic cells.

According to the histopathological findings, the present intracranial tumor is considered to have been derived from meningothelial cells, and the lesion should be diagnosed as meningioma. This diagnosis is also supported by the histopathological and immunohistochemical findings previously reported in canine meningiomas $[1,7]$. 


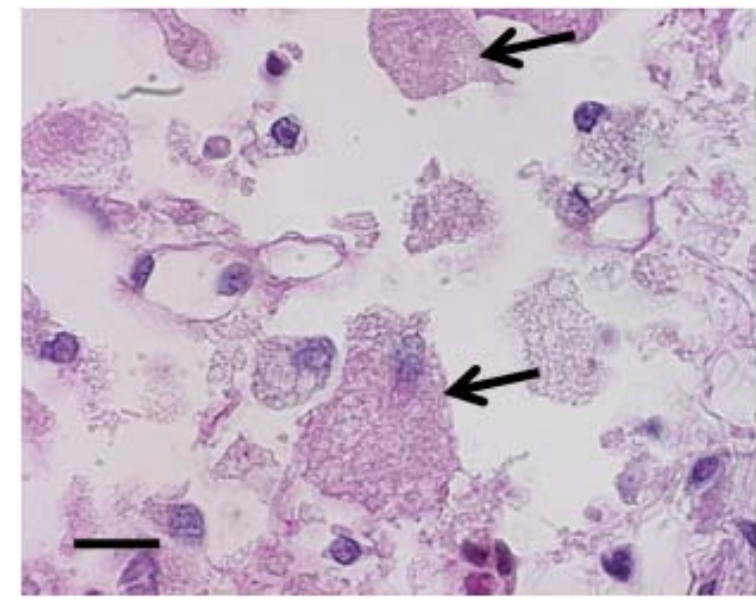

Fig. 4. Some of the neoplastic cells show weak PAS-positive granules (arrows). PAS. Bar, $50 \mu \mathrm{m}$.

The distribution of the lesions in the present case was different from that usually observed in canine meningiomas. While plaque-like masses were formed over the cerebrum in this case, canine menigiomas usually grow as well-demarcated masses $[4,13]$. This suggests that the present case had a malignant potential. Actually, neoplastic cells apparently invaded into the cerebral parenchyma. Menigiomas, except for anaplastic type, are considered to be benign in the current WHO classification [3]. However, metastatic growth into the lung and heart were seen in one case of canine meningioma with a granular cell component [10].

Large tumor cells with foamy cytoplasm were distinguished in the growth foci, and were the characterisitic feature of the cellular morphology in the present case. We consider that some degenerative effects may have influenced the cellular morphology in this case. That is, degenerative change had progressed by becoming the organelles unclearly.

As for the classification of the present canine meningioma case, granular cell meningioma would be the most suitable among the histological subtypes in the WHO classification. The description of granular cell meningioma in the classification is as follows: "A meningioma predominantly composed of oval-to-polygonal cells with abundant granular eosinophilic cytoplasm" and "The cytoplasmic granules stain positively with PAS and are resistant to digestion with diastase" [3]. In the present case, the neoplastic cells certainly contained granular eosinophilic cytoplasm, while the intensity of PAS staining for the cytoplasmic granules was very weak. The weakened PAS staining intensity might have resulted at least in part from the degenerative changes in this case. In the reported cases of canine meningioma with granular cell component, the majority of the large, polygonal cells contained variable amounts of cytoplasmic PAS-positive granules, but granules were seen only occasionally in the smaller cells [10]. Therefore, in that case, the amount of cytoplasmic granules is variably in
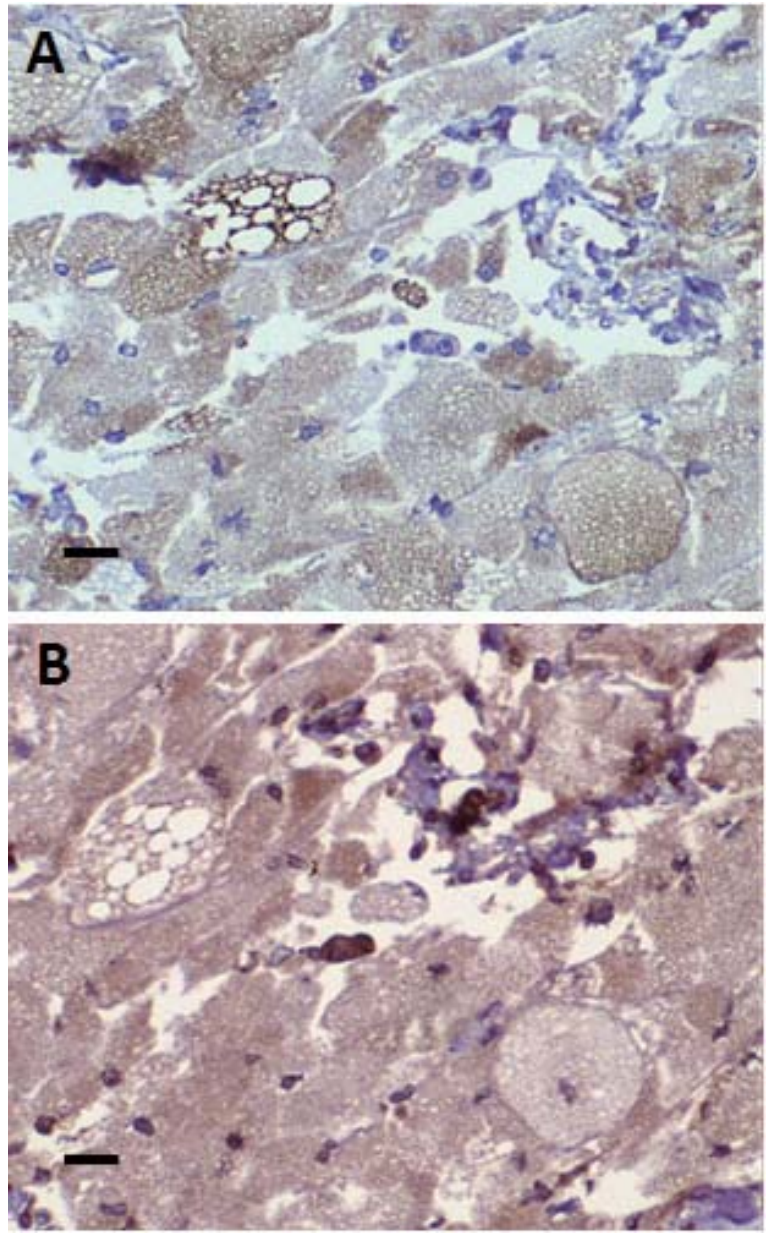

Fig. 5. Neoplastic cells are strongly positive for vimentin (A) and S-100 (B). Bar, $20 \mu \mathrm{m}$.

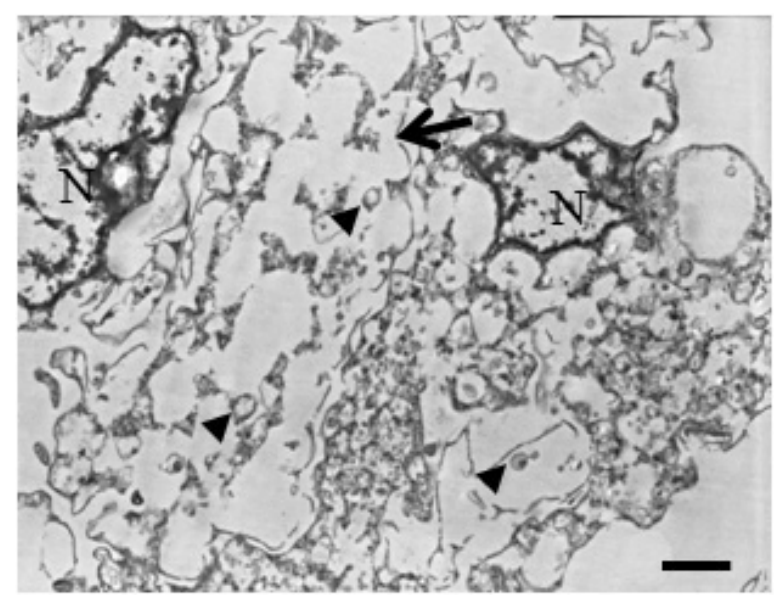

Fig. 6. Ultrastructural appearance of neoplastic cells. The cells contain vesicular structures (arrow) and small round-shaped bodies (arrowheads). N: nucleus. Bar, $2 \mu \mathrm{m}$. 
tumor cells. In this respect, that case is very similar to our present case. The similarity supports the diagnosis of this case as granular cell meningioma.

As the differential diagnosis of this case, canine meningeal granular cell tumor would be considered. As mentioned above, the histogenesis of meningioma and granular cell tumor in dogs is not considered to be the same, and the present intracranial tumor is considered to have been derived from meningothelial cells. Therefore, this case should be diagnosed as meningioma. Higgins et al. reported the ultrastructural findings of canine meningeal granular cell tumors, such as eccentric nuclei in the tumor cells and large numbers of autophagosomes containing multiple dense bodies [2], but similar features could not be observed in the present case.

We report here a case of intracranial meningioma with polygonal granular cell proliferation in a Chihuahua dog. However, ultrastructurally, typical autophagosomes were not observed in the neoplastic cells, therefore the differentiation into typical granular cells was not clear. We are considering that this case should be diagnosed as canine meningioma with granular cell appearance.

\section{REFERENCES}

1. Barnhart, K.F., Wojcieszyn, J. and Storts, R.W. 2002. Vet
Pathol. 39: 311-321.

2. Higgins, R.J., LeCouteur, R.A., Vernau, K.M., Sturges, B.K., Obradovich, J.E. and Bollen, A.W. 2001. Vet. Pathol. 38: 620 627.

3. Koestner, A., Bilzer, T., Fatzer, R., Schulman, F. Y., Summers, B. A. and Van Winkle, T. J. 1999. pp. 27-29. Armed Forces Institute of Pathology. Washington, DC.

4. Koestner, A. and Higgins, R. J. 2002. pp. 712-723. Iowa State Press. Iowa.

5. Lantos, P.L., Louis, D. N., Rosenblum, M. K. and Kleihues, P. 2002. pp. 905-926. Arnold. London.

6. Mauldin, E.A., Deehr, A.J., Hertzke, D. and Dubielzig, R.R. 2000. Vet. Ophthalmol. 3: 11-16.

7. Montoliu, P., Anor, S., Vidal, E. and Pumarola, M. 2006. $J$ Comp. Pathol. 135: 200-207.

8. Parker, G.A., Botha, W., Van Dellen, A. and Casey, H.W. 1978. Cornell. Vet. 68: 506-520.

9. Patnaik, A.K. 1993. Vet. Pathol. 30: 176-185.

10. Perez, V., Vidal, E., Gonzalez, N., Benavides, J., Ferreras, M.C., Villagrasa, M. and Pumarola, M. 2005. J. Comp. Pathol. 133: 212-217.

11. Sharkey, L.C., McDonnell, J.J. and Alroy, J. 2004. Vet. Clin. Pathol. 33: 111-114.

12. Snyder, J.M., Shofer, F.S., Van Winkle, T.J. and Massicotte, C. 2006. J. Vet. Intern. Med. 20: 669-675.

13. Summers, B.A., Cummings, J. F. and de Lahunta, A 1995. pp. 355-362. Mosby. St Louis. 